TRABAJO DE INVESTIGACIÓN

PROGRAMA DE DOCTORADO EN MEDICINA INTERNA

\title{
HOSPITAL DE DÍA DE ENFERMEDADES RESPIRATORIAS: IMPACTO SOBRE LA TASA DE INGRESOS HOSPITALARIOS POR EXACERBACIONES DE LA ENFERMEDAD PULMONAR OBSTRUCTIVA CRÓNICA
}

Autor: Luis Fernando Casas Méndez

Director: Dr. Christian Domingo Ribas

Convocatoria Septiembre 2013

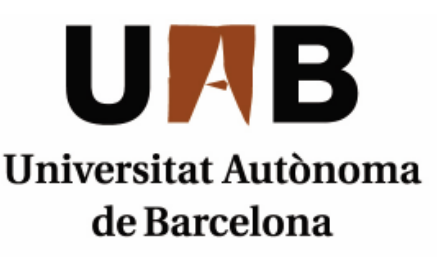

DEPARTAMENT DE MEDICINA 


\section{ÍNDEX}

Certificat del director del treball de recerca ............................................. 3

Document d'autorització per a introduir els treballs dels alumnes a

dipòsits digitals de la UAB i del CBUC ........................................................ 4

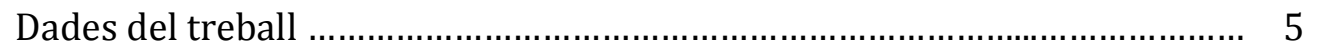

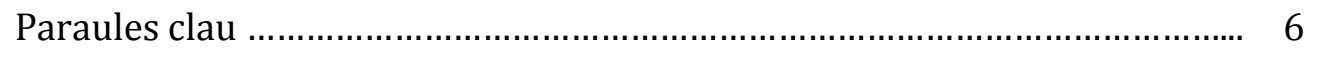

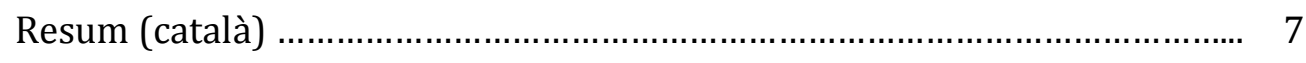

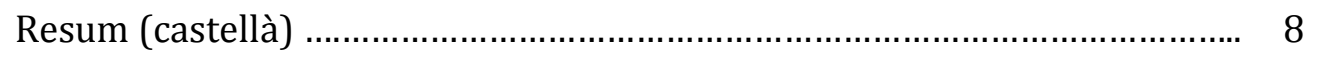

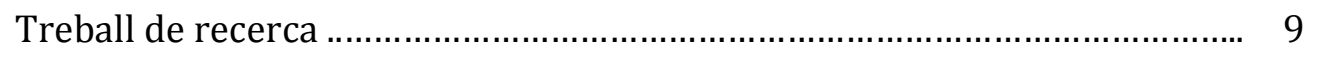

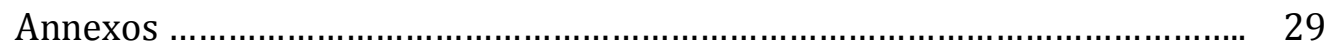




\section{CERTIFICAT DEL DIRECTOR DEL TREBALL DE RECERCA}

Christian Domingo i Ribas, Professor Associat del Departament de Medicina de la Universitat Autònoma de Barcelona,

FA CONSTAR,

Que el treball titulat

“Hospital de día de enfermedades respiratorias: impacto sobre la tasa de ingresos hospitalarios por exacerbaciones de la enfermedad pulmonar obstructiva crónica" ha estat realitzat sota la meva direcció pel llicenciat Luis Fernando Casas Méndez, trobant-se en condicions de poder ser presentat com a treball d'investigació de 12 crèdits, dins el programa de doctorat en Medicina Interna/Diagnòstic per la Imatge (Curs 2012-2013), a la convocatòria de Setembre de 2013.

Signatura

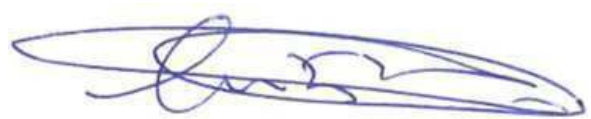

Barcelona, 5 de setembre de dos mil tretze. 


\title{
Document d'autorització per a introduir els Treballs dels alumnes a dipòsits digitals de la UAB i del CBUC
}

\author{
Nom i Cognoms d l'Autor: Luis Fernando Casas Méndez
}

NIE: X7405569-Y

Com a únic titular dels drets de propietat intel-lectual del treball: "Hospital de día de enfermedades respiratorias: impacto sobre la tasa de ingresos hospitalarios por exacerbaciones de la enfermedad pulmonar obstructiva crónica"

Autoritzo a la Universitat Autònoma de Barcelona (UAB) i al Consorci de Biblioteques Universitàries de Catalunya (CBUC) a dipositar aquest treball al Dipòsit de la Recerca de Catalunya (RecerCat) o qualsevol altre creat per la UAB o el CBUC amb les finalitats de facilitar la preservació i la difusió de la recerca i la investigació universitària.

Per tant, autoritzo a la UAB, i al CBUC a realitzar els actes que siguin necessaris per tal d'introduir el treball als esmentats dipòsits, així com per preservar-lo i donar-li accés mitjançant comunicació pública. Aquestes institucions no estan obligades a reproduir el treball en els mateixos formats o resolucions en què serà dipositat originàriament. La cessió de l'exercici dels drets necessaris per tal de realitzar totes aquestes accions es fa amb caràcter de no exclusivitat, és a dir, sóc lliure de publicar-lo a qualsevol altre lloc.

Declaro que no vulnero cap dret de tercers ja sigui de propietat intel-lectual, industrial, secret comercial o qualsevol altre, en subscriure aquesta autorització, ni en relació al contingut d'aquest treball, de manera que exonero la UAB i el CBUC de qualsevol obligació o responsabilitat davant qualsevol acció legal que es pugui suscitar derivada del treball dipositat.

Finalment declaro que accepto que des del repositori es doni accés al treball mitjançant una llicència Creative Commons, "Reconeixement-NoComercialSenseObraDerivada 2.5 Espanya" amb la qual es permet copiar, distribuir i comunicar públicament l'obra sempre que se'n citin l'autor original i la institució i no se'n faci cap ús comercial ni obra derivada.

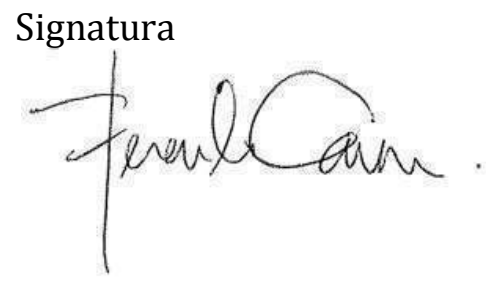

Barcelona, 5 de setembre de dos mil tretze 


\section{DADES DEL TREBALL}

\section{AUTOR}

Luis Fernando Casas Méndez

TÍTOL

Hospital de día de enfermedades respiratorias: Impacto sobre la tasa de ingresos hospitalarios por exacerbaciones de la enfermedad pulmonar obstructiva crónica.

\section{ANY D’ELABORACIÓ}

$2011-2012$

DIRECTOR DEL TREBALL

Christian Domingo i Ribas

TIPUS DE TREBALL

Treball de recerca

\section{TITULACIÓ}

Medicina

\section{DEPARTAMENT}

Medicina, Facultat de Medicina, UAB. 
PARAULES CLAU

Malaltia Pulmonar Obstructiva Crònica

Hospital de Dia

Servei d'Urgències

\section{PALABRAS CLAVE}

Enfermedad Pulmonar Obstructiva Crónica

Hospital de Día

Servicio de Urgencias 


\section{RESUM}

Les exacerbacions de la malaltia pulmonar obstructiva crònica (EA-MPOC) constitueixen una de les principals causes d'hospitalització i d'assistència als Serveis d'Urgències, la qual cosa els confereix ser les responsables d'una gran càrrega sobre el sistema sanitari. En el nostre estudi hem avaluat l'impacte d'una manera assistencial alternativa (els hospitals de dia de malalties respiratòries HDMR-) sobre la taxa d'ingressos durant les EA-MPOC. Els resultats mostren que l'atenció en els HDMR pot disminuir el nombre d'ingressos en comparació amb els Serveis d'Urgències a partir d'un model d'atenció continuada i multidimensional. 


\section{RESUMEN}

Las exacerbaciones de la enfermedad pulmonar obstructiva crónica (EA-EPOC) constituyen una de las principales causas de hospitalización y de asistencia a los Servicios de Urgencias, lo cual les confiere ser las responsables de una gran carga sobre el sistema sanitario. En nuestro estudio hemos evaluado el impacto de un modo asistencial alternativo (los hospitales de día de enfermedades respiratorias -HDER-) sobre la tasa de ingresos durante las EA-EPOC. Los resultados muestran que la atención en los HDER puede disminuir el número de ingresos en comparación con los Servicios de Urgencias a partir de un modelo de atención continuada y multidimensional. 
TREBALL DE RECERCA:

\section{HOSPITAL DE DÍA DE ENFERMEDADES RESPIRATORIAS: IMPACTO SOBRE LA TASA DE INGRESOS HOSPITALARIOS POR EXACERBACIONES DE LA ENFERMEDAD PULMONAR OBSTRUCTIVA CRÓNICA}

Med Clin (Barc). 2011;136(15):665-668 


\section{AUTORES}

\section{Luis Fernando Casas Méndez ${ }^{1}$}

Concepción Montón Soler ${ }^{1}$

Marisa Baré Mañás 2

Jordi Casabon Salas 1

Xavier Pomares Amigó ${ }^{1}$

Urko Aguirre Larracoechea ${ }^{3}$

1 Servicio de Neumología. Hospital de Sabadell, Corporació Sanitària i Universitària Parc Taulí. Universitat Autònoma de Barcelona. Sabadell, Barcelona, España.

2 Epidemiología Clínica - Cribado de Cáncer. UDIAT-CD. Hospital de Sabadell, Corporació Sanitària i Universitària Parc Taulí. Universitat Autònoma de Barcelona. Sabadell, Barcelona, España.

${ }^{3}$ Unidad de Investigación, Hospital de Galdakao-Usansolo, CIBER Epidemiología y Salud Pública (CIBERESP), Galdakao, Bizcaia, España 


\section{ABSTRACT}

Fundamento y objetivo: Las exacerbaciones de la enfermedad pulmonar obstructiva crónica (EA-EPOC) constituyen un factor determinante en el deterioro de los pacientes, con gran repercusión sobre el gasto sanitario. El objetivo de este estudio fue evaluar el impacto de la atención de las EA-EPOC en un hospital de día de enfermedades respiratorias (HDER) sobre la tasa de ingresos hospitalarios.

Pacientes y método: Durante 14 meses se recogieron prospectivamente todos los episodios de EA-EPOC atendidos en el HDER y en el Servicio de Urgencias, excluyendo a los pacientes con otra causa de agudización o necesidad de ventilación mecánica invasiva.

Resultados: Se incluyeron 226 episodios (173 pacientes, edad media [DE] de 72 [8] años). En el HDER se atendieron 121 (53,5\%). El número de ingresos en el grupo del HDER fue claramente inferior $(32,2 \%$ frente a $66,7 \%, p<0,001)$, incluso en pacientes con más ingresos el año previo. No hubo diferencias en la gravedad de la exacerbación ni en la tasa de reingresos ni de mortalidad en el seguimiento.

Conclusiones: El manejo de las EA-EPOC en el HDER parece reducir el número de ingresos hospitalarios en comparación con un Servicio de Urgencias. 


\section{INTRODUCCIÓN}

Es bien conocido el importante papel que desempeñan las exacerbaciones sobre el curso clínico de la enfermedad pulmonar obstructiva crónica (EPOC), de modo que son consideradas como un factor independiente en el deterioro funcional y de la calidad de vida de los pacientes (1). Así mismo, desde el punto de vista de la gestión de recursos hospitalarios su impacto es tal, que contabilizan entre un 1 y $2 \%$ de todas las visitas a los Servicios de Urgencias (2) y casi un $10 \%$ de los ingresos en salas de hospitalización médica (3).

Precisamente, en la búsqueda de nuevas alternativas para el manejo de los pacientes con EPOC, varios estudios han demostrado la efectividad y eficiencia de las consultas monográficas (principalmente sobre el gasto sanitario) y los beneficios de programas de atención continuada, como los ofrecidos por los hospitales de día (HD), los cuales han permitido disminuir el número de asistencias a urgencias y el de ingresos hospitalarios anuales de cada paciente (en relación con el año anterior) (4-6).

A pesar de que en Cataluña más de una tercera parte de los hospitales cuentan con HD de enfermedades respiratorias (HDER) (6), en la literatura médica no existen datos acerca de su repercusión sobre los ingresos hospitalarios por exacerbaciones de la EPOC (EA-EPOC) en comparación con un Servicio de Urgencias médicas. 
El objetivo principal de nuestro estudio ha sido establecer el impacto de la atención de las EA-EPOC en un HDER sobre la tasa de ingresos hospitalarios, con respecto a la asistencia convencional en un Servicio de Urgencias. 


\section{PACIENTES Y MÉTODOS}

Desde octubre de 2007 y durante 14 meses se recogieron prospectivamente todos los episodios de EA-EPOC, definida según la normativa de la Sociedad Española de Neumología y Cirugía torácica (SEPAR) (7), atendidos en el hospital de día de enfermedades respiratorias (grupo HDER) y en el Servicio de Urgencias (grupo UCIAS) de un hospital universitario. El comité de ética del hospital aprobó la realización del estudio y todos los pacientes dieron su consentimiento informado.

Se excluyeron los pacientes con otra causa identificable de empeoramiento clínico o agudización (neumonía, embolia pulmonar, insuficiencia cardiaca izquierda, arritmia grave y neumotórax) y aquellos con necesidad de ventilación mecánica invasiva. Se registraron las principales variables relacionadas con la gravedad de la EPOC (porcentaje del $\mathrm{FEV}_{1}$-volumen espiratorio forzado en el primer segundo- en fase estable, tratamiento con oxigenoterapia crónica domiciliaria y número de ingresos hospitalarios por EA-EPOC durante el año anterior) y de la EA-EPOC (valor de presión parcial de oxígeno en sangre arterial corregida por la fracción inspirada de oxígeno -cociente $\mathrm{P}_{\mathrm{a}} \mathrm{O} 2 / \mathrm{F}_{\mathrm{i}} \mathrm{O}_{2}$ - y presencia de acidosis respiratoria con $\mathrm{pH} \leq 7,30$ ), el destino del paciente y la tasa de reingresos y de mortalidad a los 2 meses de seguimiento.

Como destino al alta de urgencias y del HDER fueron consideradas tres posibilidades después de la atención de la EA-EPOC: ingreso en la sala de hospitalización convencional (definido como una estancia mayor a 48 horas en 
planta de neumología, medicina interna o unidad de corta estancia); domicilio del paciente (con control por médico de cabecera o especialista habitual) y Unidad de Hospitalización Domiciliaria (UHD). Esta última es un dispositivo que tiene la disponibilidad y los recursos para administrar tratamientos endovenosos, medicación nebulizada y oxigenoterapia con supervisión médica y de enfermería estricta en el domicilio del paciente.

\section{Análisis estadístico}

Se llevó a cabo un análisis descriptivo de todas las variables sociodemográficas, de la situación basal del paciente y de la gravedad de la EA-EPOC. Se realizó un análisis bivariante para comparar las mismas variables según fueran atendidos en HDER o en UCIAS, así como según el destino final al alta (ingreso hospitalario frenta a no ingreso). Para esto último, se aplicaron técnicas bivariantes de regresión logística basadas en ecuaciones de estimación generalizadas (GEE), que tienen en cuenta la posible correlación intraindividuos. Finalmente, se aplicaron técnicas multivariadas basadas en GEE (método enter) para identificar el efecto independiente de las variables significativas en el análisis bivariante sobre la probabilidad de ingreso hospitalario, ajustando por edad, y se estimó el área bajo la curva ROC (receiver operating characteristic). Para todas las estimaciones se consideró un riesgo $\alpha$ del 5\%. Se estimaron las Odds Ratio (OR) y sus intervalos de confianza al 95\% (IC 95\%). 


\section{RESULTADOS}

Se evaluaron de forma prospectiva 226 episodios de EA-EPOC en 173 pacientes (1,30 episodios/paciente) con una edad media (DE) de 72 (8) años. De todos los episodios, 121 se atendieron en el HDER (53,5\%). En este grupo y con respecto a UCIAS, destacaba un número superior de episodios en situación de insuficiencia respiratoria crónica (todos ellos portadores de oxigenoterapia crónica domiciliaria) y que habían requerido más ingresos por EA-EPOC en el año previo al episodio estudiado. En cuanto a la gravedad de la EA-EPOC, evaluada en función de la presencia de acidosis respiratoria y de la intensidad de la hipoxemia, no se encontraron diferencias estadísticamente significativas entre los dos grupos (tabla 1).

En lo referente al destino de los episodios $(n=226)$ después de la atención de la exacerbación (fig. 1), la tasa de ingresos hospitalarios de los episodios atendidos en el HDER fue significativamente menor que en el grupo de urgencias $(32,2 \%$ frente a 66,7\%, p < 0,001), a expensas de más altas al domicilio $(49,6 \%$ frente a $23,8 \%)$ y a la UHD $(18,2 \%$ frente a $9,5 \%)$. Durante el seguimiento a dos meses no se observaron diferencias significativas en el porcentaje de reingresos $(25,8 \%$ frente a $23,8 \%, p=0,723)$ ni en la mortalidad $(1,7 \%$ frente a $4,8 \%, p=0,262)$.

El análisis multivariado ajustado por la edad mostró como único factor de riesgo asociado de forma independiente al ingreso en el hospital el tener $\geq 2$ hospitalizaciones en el año previo por EA-EPOC ( $\beta$ 1,48, IC 95\% 0,75-2,22; OR 4,40, IC 95\% 2,11-9,19), mientras que la atención de la exacerbación en el HDER 
resultó ser un factor protector del ingreso hospitalario ( $\beta-2,10$, IC 95\% -2,80- 1,39; OR 0,12, IC 95\% 0,06-0,25). El área bajo la curva ROC del modelo fue 0,75 (IC 95\% 68,7-81,3). 


\section{DISCUSIÓN}

Nuestro estudio pone de manifiesto el gran impacto que conlleva la atención en un HDER de los pacientes con EA-EPOC, pues se puede reducir la probabilidad de ingreso hospitalario entre un $75 \%$ y un $94 \%$, sin que esto se asocie con un aumento significativo de la tasa de reingresos ni de la mortalidad. Igualmente, los resultados demuestran que dicha disminución ocurre incluso en pacientes que han requerido un número superior de ingresos hospitalarios por EA-EPOC en el año previo al episodio estudiado. Esto último es de gran trascendencia, teniendo en cuenta que dicho factor se considera como explicativo independiente de la necesidad de ingreso hospitalario por una nueva exacerbación, tal y como demostró el estudio EFRAM (8). El citado estudio EFRAM también incluía otras variables que finalmente fueron predictoras de una mayor tasa de ingresos por EA-EPOC, destacando el porcentaje del valor predicho de $\mathrm{FEV}_{1}$ y la adecuación de la prescripción de oxigenoterapia crónica domiciliaria (OCD). En nuestro estudio no encontramos diferencias en la gravedad basal de la EPOC entre los dos grupos, pero sí en cuanto al porcentaje de pacientes portadores de OCD, sin poder evaluarse la adecuación de la misma.

Los HD fueron creados a partir de la necesidad de ofrecer tratamientos y procedimientos diagnósticos frecuentes a pacientes con diferentes patologías que así lo precisaran. En nuestro caso, el HDER surgió con la intención de proporcionar a los pacientes con EPOC grave un mecanismo de atención periódica apoyado en un programa de atención continuada (PAC) con el objetivo de reducir las exacerbaciones y sus consecuencias sobre el curso de la 
enfermedad. Dicho programa, entre otros, incluye: (a) control médico periódico, (b) control preferente tras las exacerbaciones u hospitalización, (c) educación sanitaria, (d) programa de fisioterapia y (e) deshabituación tabáquica. Así mismo, en el caso específico de los pacientes con EPOC grave o muy grave (estadios GOLD III-IV), el principal criterio de inclusión en el PAC es la presencia de 2 o más exacerbaciones de la enfermedad en el último año que hayan requerido ingreso hospitalario o atención en urgencias (6). La inclusión, control, educación y seguimiento de este grupo de pacientes ha permitido alcanzar importantes índices de control de la enfermedad y de conocimiento de los mismos, tanto desde el punto de vista clínico (situación basal, perfil psicológico y de cumplimiento) como sociofamiliar, favoreciendo que en las exacerbaciones sea posible establecer, considerando dichos factores, quiénes son tributarios de tratamiento ambulatorio o en régimen de hospitalización domiciliaria o bien de ingreso en planta convencional.

Otro de los resultados destacables de nuestro trabajo es la hospitalización domiciliaria de los pacientes con EA-EPOC, una alternativa altamente evaluada y reconocida, y con un claro impacto económico (reducción de costes) y sobre la dinámica hospitalaria (disponibilidad de camas), sin comprometer la satisfacción ni la garantía del cuidado del paciente (9-10). En nuestro estudio se observa una diferencia claramente significativa en la utilización de esta modalidad de tratamiento domiciliario en el grupo de pacientes atendidos en el HDER, lo cual podría explicarse por una elección de los pacientes candidatos basada en un mayor conocimiento de las condiciones domiciliarias y del cumplimiento ambulatorio. 
Entre las limitaciones del estudio, habría que destacar que no se puede descartar completamente que no exista un sesgo en la selección de los pacientes. Sin embargo, en lo referente a las características basales, ambos grupos eran comparables en cuanto a la comorbilidad (índice de Charlson) y a la gravedad de la EPOC en situación de estabilidad (medida por el $\mathrm{FEV}_{1}$ ) y, de hecho, los resultados de las otras variables evaluadas muestran en cualquier caso mayor gravedad en los pacientes atendidos en el HDER (mayor número de ingresos en el año previo y número superior de pacientes en situación de insuficiencia respiratoria crónica).

De la misma manera, en lo relacionado con la gravedad de la exacerbación, tampoco se encontraron diferencias entre los dos grupos, aunque cabe tener en consideración que actualmente no se dispone de una escala bien definida para establecer la gravedad en las EA-EPOC. Así mismo, a lo largo de todo el estudio fue difícil evaluar datos objetivos inherentes a la EA-EPOC en todos los pacientes (por ejemplo frecuencia respiratoria y gasometría arterial). De todas formas, creemos que la realización de un análisis multivariado permite ajustar las diferencias entre los dos grupos.

Finalmente, otro de los elementos a tener en cuenta es que el ámbito donde se atiende al paciente (HDER o UCIAS) influya por sí mismo, por su propio modelo organizativo, en el destino final del paciente. 
En conclusión, la atención de los pacientes con EA-EPOC en un HDER por un equipo médico y de enfermería y fisioterapia, que además los controla de forma habitual en situación estable, parece reducir el número de ingresos hospitalarios en comparación con la atención convencional en un Servicio de Urgencias. 


\section{FINANCIACIÓN}

Este trabajo ha sido financiado parcialmente por el Fondo de Investigación Sanitaria (FIS 06/90282, FIS 06/0326). 


\section{CONFLICTO DE INTERESES}

Los autores declaran no tener ningún conflicto de intereses. 


\section{AGRADECIMIENTOS}

Este trabajo ha sido posible gracias a la inestimable colaboración de la Sra. Nuria Torà, y de todo el personal de enfermería del Hospital de día de Neumología y del

Servicio de Urgencias del Hospital de Sabadell, a quienes agradecemos su inagotable capacidad de trabajo y generosidad. 


\section{REFERENCIAS}

1. Wang Q, Bourbeau J. Outcomes and health-related quality of life following hospitalization for an acute exacerbation of COPD. Respirology 2005;10:33440.

2. Otero González I, Blanco Aparicio M, Montero Martínez C, Valiño López P, Verea Hernando H. Características epidemiológicas de las exacerbaciones por EPOC y asma en un hospital general. Arch Bronconeumol. 2002;38:256-62.

3. Miravitlles M, Figueras M. El coste de la enfermedad pulmonar obstructiva crónica en España. Opciones para una optimización de recursos. Arch Bronconeumol. 2001;37:388-93.

4. Domingo Ch, Sans-Torres J, Solà J, Espuelas H, Marín A. Efectividad y eficiencia de una consulta monográfica hospitalaria para pacientes con EPOC e insuficiencia respiratoria. Arch Bronconeumol. 2006;42:104-12.

5. Solera JJ, Martínez-García MA, Román P, Orero R, Terrazas S, MartínezPechuán A. Eficacia de un programa específico para pacientes con EPOC que presentan frecuentes agudizaciones. Arch Bronconeumol. 2006;42:501-8

6. Pomares Amigó X, Montón Soler C. Hospitales de día de enfermedades respiratorias: ¿qué hemos aprendido? Med Clin (Barc). 2009. doi:10.1016/j.medcli.2009.07.028. 
7. Peces-Barba G, Barberà JA, Agustí A, Casanova C, Casas A, Izquierdo JL, et al. Guía clínica SEPAR-ALAT de diagnóstico y tratamiento de la EPOC. Arch Bronconeumol. 2008;44:271-81.

8. García-Aymerich J, Monsó E, Marrades RM, Escarrabill J, Félez MA, Sunyer J, et al. Risk factors for hospitalization for a chronic obstructive pulmonary disease exacerbation. EFRAM Study. Am J Respir Crit Care Med. 2001;164:1002-7.

9. Puig-Junoy J, Casas A, Font-Planells J, Escarrabill J, Hernández C, Alonso J, et al. The impact of home hospitalization on healthcare costs of exacerbations in COPD patients. Eur J Health Econ. 2007;8:325-32.

10. Díaz Lobato S, González Lorenzo F, Gómez Mendieta MA, Mayorales Alises S, Martín Arechabala I, Villasante Fernández-Montes C. Evaluación de un programa de hospitalización domiciliaria en pacientes con EPOC agudizada. Arch Bronconeumol. 2005;41:5-10. 
TABLA 1. Variables demográficas y clínicas de los episodios según el ámbito de atención.

\begin{tabular}{|c|c|c|c|c|c|c|}
\hline \multicolumn{3}{|c|}{ Variable } & TOTAL & HDER & UCIAS & $\mathbf{P}$ \\
\hline \multirow{3}{*}{ Demográficas } & \multicolumn{2}{|c|}{ Episodios (n) } & 226 & 121 & 105 & - \\
\hline & \multicolumn{2}{|l|}{ + $/ \sigma^{\lambda}$} & $4 / 222$ & $0 / 121$ & $4 / 101$ & - \\
\hline & \multicolumn{2}{|c|}{$\begin{array}{l}\text { Edad media en años (mediana) } \\
\text { (DE) }\end{array}$} & $72,4(72)(8)$ & $70,5(71)(6,7)$ & $75(76)(8,1)$ & 0,083 \\
\hline \multirow{6}{*}{$\begin{array}{l}\text { Situación } \\
\text { basal }\end{array}$} & \multicolumn{2}{|c|}{$\mathrm{FEV}_{1}$ medio en $\%$ (mediana) (DE) } & $\begin{array}{c}35,9(35) \\
(13,9)\end{array}$ & $\begin{array}{c}34,3(33) \\
(13,2)\end{array}$ & $\begin{array}{c}39,6(39) \\
(15,3)\end{array}$ & 0,207 \\
\hline & \multicolumn{2}{|c|}{ OCD $(n, \%)$} & $82(36,8)$ & $52(43,3)$ & $30(29,1)$ & 0,037 \\
\hline & \multirow{2}{*}{$\begin{array}{l}\text { Ingresos por EA- } \\
\text { EPOC en el último } \\
\text { año }(n, \%)\end{array}$} & $\begin{array}{c}\leq 1 \\
(\mathrm{n}, \%)\end{array}$ & $126(56,8)$ & $54(45,4)$ & $72(69,9)$ & \multirow{2}{*}{0,004} \\
\hline & & $\begin{array}{c}\geq 2 \\
(\mathrm{n}, \%)\end{array}$ & $96(43,2)$ & $65(54,6)$ & $31(30,1)$ & \\
\hline & \multirow{2}{*}{$\begin{array}{l}\text { Índice de Charlson } \\
(\mathrm{n}, \%)\end{array}$} & $\begin{array}{l}1,2 \text { puntos } \\
\text { (n, \%) }\end{array}$ & $173(76,5)$ & $99(81,8)$ & $74(70,5)$ & \multirow{2}{*}{0,210} \\
\hline & & $\begin{array}{c}\geq 3 \text { puntos } \\
\quad(n, \%)\end{array}$ & $53(23,5)$ & $22(18,2)$ & $31(29,5)$ & \\
\hline \multirow{2}{*}{$\begin{array}{l}\text { Gravedad de } \\
\text { la EA-EPOC }\end{array}$} & \multicolumn{2}{|c|}{$\begin{array}{c}\mathrm{PaO}_{2} / \mathrm{F}_{\mathrm{i}} \mathrm{O}_{2} \text { media en mmHg } \\
\text { (mediana) (DE) }\end{array}$} & $\begin{array}{c}250,5 \\
(252,4)(54,5)\end{array}$ & $\begin{array}{c}249,2 \\
(252,4)(43,4)\end{array}$ & $\begin{array}{c}260,4 \\
(253,8)(88,7)\end{array}$ & 0,460 \\
\hline & \multicolumn{2}{|c|}{ Acidosis respiratoria (n, \%) } & $7(4,5)$ & $2(2,9)$ & $5(5,8)$ & 0,292 \\
\hline \multicolumn{7}{|c|}{$\begin{array}{l}\text { HDER: Hospital de día de enfermedades respiratorias; UCIAS: Urgencias; DE: Desviación Estándar; } \mathrm{FEV}_{1} \text { : Volumen Espiratorio } \\
\text { Forzado en el primer segundo (en \% del valor de referencia); OCD: Oxigenoterapia Crónica Domiciliaria; EA-EPOC: } \\
\text { Exacerbación de la Enfermedad Pulmonar Obstructiva Crónica; } \mathrm{P}_{\mathrm{a}} \mathrm{O}_{2} \text { : Presión parcial de oxígeno en sangre arterial; } \mathrm{F}_{\mathrm{i}} \mathrm{O}_{2} \text { : } \\
\text { Fracción inspirada de oxígeno }\end{array}$} \\
\hline
\end{tabular}


FIGURA 1. Destino final de los pacientes atendidos por exacerbación de la enfermedad pulmonar obstructiva crónica.

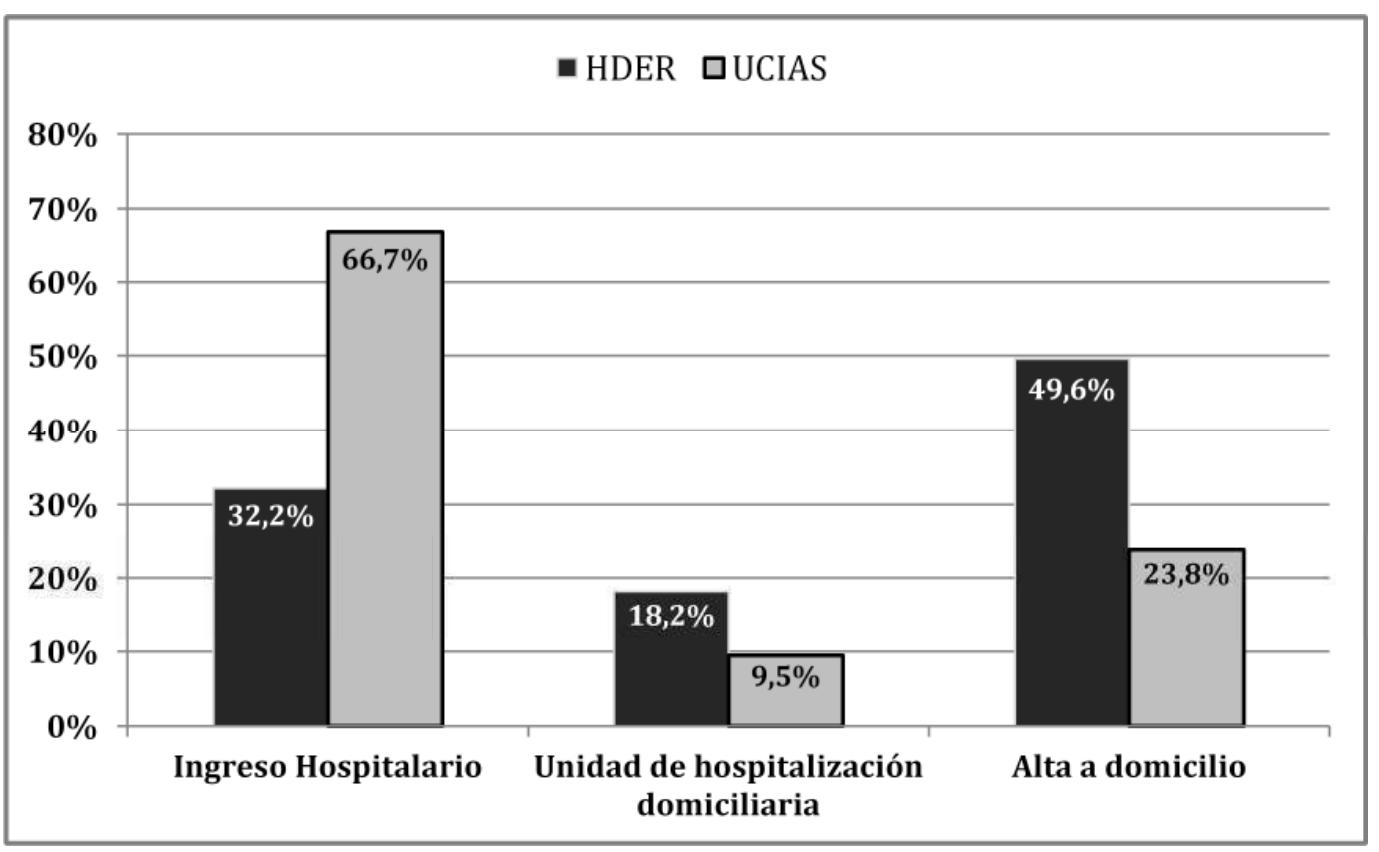


ANNEXOS 
Original breve

\title{
Hospital de día de enfermedades respiratorias: impacto sobre la tasa de ingresos hospitalarios por exacerbaciones de la enfermedad pulmonar obstructiva crónica
}

\author{
Luis Fernando Casas Méndez ${ }^{\mathrm{a}, *}$, Concepción Montón Soler ${ }^{\mathrm{a}}$, Marisa Baré Mañas ${ }^{\mathrm{b}}$, Jordi Casabon Salas ${ }^{\mathrm{a}}$, \\ Xavier Pomares Amigó ${ }^{a}$ y Urko Aguirre Larracoechea ${ }^{c}$ \\ a Servicio de Neumología, Hospital de Sabadell, Corporació Sanitària i Universitària Parc Taulí, Universitat Autònoma de Barcelona, Sabadell, Barcelona, España \\ ${ }^{\mathrm{b}}$ Epidemiología Clínica-Cribado de Cáncer. UDIAT-CD. Hospital de Sabadell, Corporació Sanitària i Universitària Parc Tauli, Universitat Autònoma de Barcelona, Sabadell, Barcelona, España \\ ${ }^{c}$ Unidad de Investigación, Hospital de Galdakao-Usansolo, CIBER Epidemiología y Salud Pública (CIBERESP), Galdakao, Bizcaia, España
}

INFORMACIÓN DEL ARTÍCULO

\section{Historia del artículo:}

Recibido el 23 de marzo de 2010

Aceptado el 27 de julio de 2010

On-line el 16 de marzo de 2011

\section{Palabras clave:}

Enfermedad pulmonar obstructiva crónica Hospital de día

Servicio de Urgencias

\section{R E S U M E N}

Fundamento y objetivo: Las exacerbaciones de la enfermedad pulmonar obstructiva crónica (EA-EPOC) constituyen un factor determinante en el deterioro de los pacientes, con gran repercusión sobre el gasto sanitario. El objetivo de este estudio fue evaluar el impacto de la atención de las EA-EPOC en un hospital de día de enfermedades respiratorias (HDER) sobre la tasa de ingresos hospitalarios.

Pacientes y método: Durante 14 meses se recogieron prospectivamente todos los episodios de EA-EPOC atendidos en el HDER y en el Servicio de Urgencias, excluyendo a los pacientes con otra causa de agudización o necesidad de ventilación mecánica invasiva.

Resultados: Se incluyeron 226 episodios (173 pacientes, edad media [DE] de 72 [8] años). En el HDER se atendieron 121 (53,5\%). El número de ingresos en el grupo del HDER fue claramente inferior (32,2 frente a $66,7 \%, \mathrm{p}<0,001)$, incluso en pacientes con más ingresos el año previo. No hubo diferencias en la gravedad de la exacerbación ni en la tasa de reingresos ni de mortalidad en el seguimiento.

Conclusiones: El manejo de las EA-EPOC en el HDER parece reducir el número de ingresos hospitalarios en comparación con un Servicio de Urgencias.

(c) 2010 Elsevier España, S.L. Todos los derechos reservados.

\section{Respiratory day hospital: Impact on the rate of hospital admissions due to chronic obstructive pulmonary disease exacerbations}

\begin{abstract}
A B S T R A C T
Background and objective: Acute exacerbations of chronic obstructive pulmonary disease (AE-COPD) are considered to be the most important factor in determining the course of the disease, and have great impact on the health care system. We evaluated the effects of a Respiratory Day Hospital (RDH) on the rate of hospital admissions due to AE-COPD.

Patients and methods: During 14 months, every case of AE-COPD seen at the RDH and emergency services was recruited prospectively. Patients were excluded when another known cause of exacerbation was present and/or when they required invasive mechanical ventilation.

Results: 226 episodes (173 patients) were included (72, SD 8 years old). One hundred and twenty one cases received treatment at the $\mathrm{RDH}$ (53.5\%). Admissions from the RDH group were noticeably lower ( 32.2 vs. $66.7 \%, \mathrm{p}<0.001$ ), even in patients with more admissions due to AE-COPD the year before the study. There were no differences either in terms of AE-COPD severity or in readmissions and mortality rates during the follow up.

Conclusions: Management of AE-COPD in a RDH seems to be associated with a lower rate of hospital admissions in comparison with an emergency department.
\end{abstract}

(c) 2010 Elsevier España, S.L. All rights reserved.

\footnotetext{
* Autor para correspondencia.

Correo electrónico: Icasas@tauli.cat (L.F. Casas Méndez).
} 


\section{Introducción}

Es bien conocido el importante papel que desempeñan las exacerbaciones sobre el curso clínico de la enfermedad pulmonar obstructiva crónica (EPOC), de modo que son consideradas como un factor independiente en el deterioro funcional y de la calidad de vida de los pacientes ${ }^{1}$. Así mismo, desde el punto de vista de la gestión de recursos hospitalarios, su impacto es tal, que contabilizan entre un 1 y $2 \%$ de todas las visitas a los Servicios de Urgencias ${ }^{2}$ y casi un $10 \%$ de los ingresos en salas de hospitalización médica ${ }^{3}$.

Precisamente, en la búsqueda de nuevas alternativas para el manejo de los pacientes con EPOC, varios estudios han demostrado la efectividad y eficiencia de las consultas monográficas (principalmente sobre el gasto sanitario) y los beneficios de programas de atención continuada, como los ofrecidos por los hospitales de día (HD), los cuales han permitido disminuir el número de asistencias a urgencias y el de ingresos hospitalarios anuales de cada paciente (en relación con el año anterior) ${ }^{4-6}$.

A pesar de que en Cataluña más de una tercera parte de los hospitales cuentan con HD de enfermedades respiratorias $(\mathrm{HDER})^{6}$, en la literatura médica no existen datos acerca de su repercusión sobre los ingresos hospitalarios por exacerbaciones de la EPOC (EA-EPOC) en comparación con un Servicio de Urgencias médicas.

El objetivo principal de nuestro estudio ha sido establecer el impacto de la atención de las EA-EPOC en un HDER sobre la tasa de ingresos hospitalarios, con respecto a la asistencia convencional en un Servicio de Urgencias.

\section{Pacientes y métodos}

Desde octubre de 2007 y durante 14 meses se recogieron prospectivamente todos los episodios de EA-EPOC, definida según la normativa de la Sociedad Española de Neumología y Cirugía torácica $(\mathrm{SEPAR})^{7}$, atendidos en el hospital de día de enfermedades respiratorias (grupo HDER) y en el Servicio de Urgencias (grupo UCIAS) de un hospital universitario. El comité de ética del hospital aprobó la realización del estudio y todos los pacientes dieron su consentimiento informado.

Se excluyeron los pacientes con otra causa identificable de empeoramiento clínico o agudización (neumonía, embolia pulmonar, insuficiencia cardiaca izquierda, arritmia grave y neumotórax) y aquellos con necesidad de ventilación mecánica invasiva. Se registraron las principales variables relacionadas con la gravedad de la EPOC (porcentaje del $\mathrm{FEV}_{1}$-volumen espiratorio forzado en el primer segundo- en fase estable, tratamiento con oxigenoterapia crónica domiciliaria y número de ingresos hospitalarios por EA-EPOC durante el año anterior) y de la EA-EPOC (valor de presión parcial de oxígeno en sangre arterial corregida por la fracción inspirada de oxígeno -cociente $\mathrm{P}_{\mathrm{a}} \mathrm{O}_{2} / \mathrm{F}_{\mathrm{i}} \mathrm{O}_{2}$ - y presencia de acidosis respiratoria con $\mathrm{pH} \leq 7,30$ ), el destino del paciente y la tasa de reingresos y de mortalidad a los 2 meses de seguimiento.

Como destino al alta de urgencias y del HDER fueron consideradas tres posibilidades después de la atención de la EAEPOC: ingreso en la sala de hospitalización convencional (definido como una estancia mayor a 48 horas en planta de neumología, medicina interna o unidad de corta estancia); domicilio del paciente (con control por médico de cabecera o especialista habitual); y Unidad de Hospitalización Domiciliaria (UHD). Esta última es un dispositivo que tiene la disponibilidad y los recursos para administrar tratamientos endovenosos, medicación nebulizada y oxigenoterapia con supervisión médica y de enfermería estricta en el domicilio del paciente.

\section{Análisis estadístico}

Se llevó a cabo un análisis descriptivo de todas las variables sociodemográficas, de la situación basal del paciente y de la gravedad de la EA-EPOC. Se realizó un análisis bivariante para comparar las mismas variables según fueran atendidos en HDER o en UCIAS, así como según el destino final al alta (ingreso hospitalario frente a no ingreso). Para esto último, se aplicaron técnicas bivariantes de regresión logística basadas en ecuaciones de estimación generalizadas (GEE), que tienen en cuenta la posible correlación intraindividuos. Finalmente, se aplicaron técnicas multivariadas basadas en GEE (método enter) para identificar el efecto independiente de las variables significativas en el análisis bivariante sobre la probabilidad de ingreso hospitalario, ajustando por edad, y se estimó el área bajo la curva ROC (receiver operating characteristic). Para todas las estimaciones se consideró un riesgo $\alpha$ del 5\%. Se estimaron las Odds Ratio (OR) y sus intervalos de confianza del 95\% (IC 95\%).

\section{Resultados}

Se evaluaron de forma prospectiva 226 episodios de EA-EPOC en 173 pacientes (1,30 episodios/paciente) con una edad media (DE) de 72 (8) años. De todos los episodios, 121 se atendieron en el HDER (53,5\%). En este grupo y con respecto a UCIAS, destacaba un número superior de episodios en situación de insuficiencia respiratoria crónica (todos ellos portadores de oxigenoterapia crónica domiciliaria) y que habían requerido más ingresos por EAEPOC en el año previo al episodio estudiado. En cuanto a la gravedad de la EA-EPOC, evaluada en función de la presencia de acidosis respiratoria y de la intensidad de la hipoxemia, no se encontraron diferencias estadísticamente significativas entre los dos grupos (tabla 1).

En lo referente al destino de los episodios $(n=226)$ después de la atención de la exacerbación (fig. 1), la tasa de ingresos hospitalarios de los episodios atendidos en el HDER fue significativamente menor que en el grupo de urgencias (32,2 frente a $66,7 \%, \mathrm{p}<0,001$ ), a expensas de más altas al domicilio (49,6 frente a $23,8 \%$ ) y a la UHD (18,2 frente a $9,5 \%$ ). Durante el seguimiento a dos meses no se observaron diferencias significativas en el porcentaje de reingresos $(25,8$ frente a $23,8 \%, p=0,723)$ ni en la mortalidad $(1,7$ frente a $4,8 \%, \mathrm{p}=0,262)$.

El análisis multivariado ajustado por la edad mostró como único factor de riesgo asociado de forma independiente al ingreso en el hospital el tener $\geq 2$ hospitalizaciones en el año previo por EAEPOC ( $\beta$ 1,48, IC 95\% 0,75-2,22; OR 4,40, IC 95\% 2,11-9,19), mientras que la atención de la exacerbación en el HDER resultó ser un factor protector del ingreso hospitalario ( $\beta-2,10$, IC 95\% - 2,80$-1,39$; OR 0,12 , IC $95 \%$ 0,06-0,25). El área bajo la curva ROC del modelo fue 0,75 (IC 95\% 68,7-81,3).

\section{Discusión}

Nuestro estudio pone de manifiesto el gran impacto que conlleva la atención en un HDER de los pacientes con EA-EPOC, pues se puede reducir la probabilidad de ingreso hospitalario entre un 75 y un $94 \%$, sin que esto se asocie con un aumento significativo de la tasa de reingresos ni de la mortalidad. Igualmente, los resultados demuestran que dicha disminución ocurre incluso en pacientes que han requerido un número superior de ingresos hospitalarios por EA-EPOC en el año previo al episodio estudiado. Esto último es de gran trascendencia, teniendo en cuenta que dicho factor se considera como explicativo independiente de la necesidad de ingreso hospitalario por una nueva exacerbación, tal como demostró el estudio EFRAM $^{8}$. El citado estudio EFRAM también incluía otras variables que finalmente fueron predictoras de una 
Tabla 1

Variables demográficas y clínicas en cada episodio según el ámbito de atención

\begin{tabular}{|c|c|c|c|c|}
\hline Variable & Total & HDER & UCIAS & $\mathrm{p}$ \\
\hline \multicolumn{5}{|l|}{ Demográficas } \\
\hline Episodios (n) & 226 & 121 & 105 & - \\
\hline भ/ & $4 / 222$ & $0 / 121$ & $4 / 101$ & - \\
\hline Edad media en años (mediana) (DE) & $72,4(72)(8)$ & $70,5(71)(6,7)$ & $75(76)(8,1)$ & 0,083 \\
\hline \multicolumn{5}{|l|}{ Situación basal } \\
\hline$F E V_{1}$ medio en $\%$ (mediana) (DE) & $35,9(35)(13,9)$ & $34,3(33)(13,2)$ & $39,6(39)(15,3)$ & 0,207 \\
\hline$O C D(n, \%)$ & $82(36,8)$ & $52(43,3)$ & $30(29,1)$ & 0,037 \\
\hline \multicolumn{5}{|l|}{ Ingresos por EA-EPOC en el último año (n, \%) } \\
\hline$\leq 1(n, \%)$ & $126(56,8)$ & $54(45,4)$ & $72(69,9)$ & 0,004 \\
\hline$\geq 2(\mathrm{n}, \%)$ & $96(43,2)$ & $65(54,6)$ & $31(30,1)$ & \\
\hline \multicolumn{5}{|l|}{ Indice de Charlson ( $n, \%)$} \\
\hline 1,2 puntos $(n, \%)$ & $173(76,5)$ & $99(81,8)$ & $74(70,5)$ & 0,210 \\
\hline$\geq 3$ puntos $(n, \%)$ & $53(23,5)$ & $22(18,2)$ & $31(29,5)$ & \\
\hline \multicolumn{5}{|l|}{ Gravedad de la EA-EPOC } \\
\hline $\mathrm{P}_{a} \mathrm{O}_{2} / \mathrm{F}_{i} \mathrm{O}_{2}$ media en $\mathrm{mmHg}$ (mediana) (DE) & $250,5(252,4)(54,5)$ & $249,2(252,4)(43,4)$ & $260,4(253,8)(88,7)$ & 0,460 \\
\hline Acidosis respiratoria $(n, \%)$ & $7(4,5)$ & $2(2,9)$ & $5(5,8)$ & 0,292 \\
\hline
\end{tabular}

DE: desviación estándar; EA-EPOC: exacerbación de la enfermedad pulmonar obstructiva crónica; HDER: hospital de día de enfermedades respiratorias; FEV ${ }_{1}$ : volumen

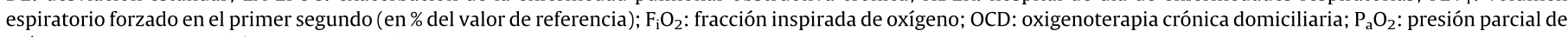
oxígeno en sangre arterial; UCIAS: Urgencias.

HDER $\square$ UCIAS

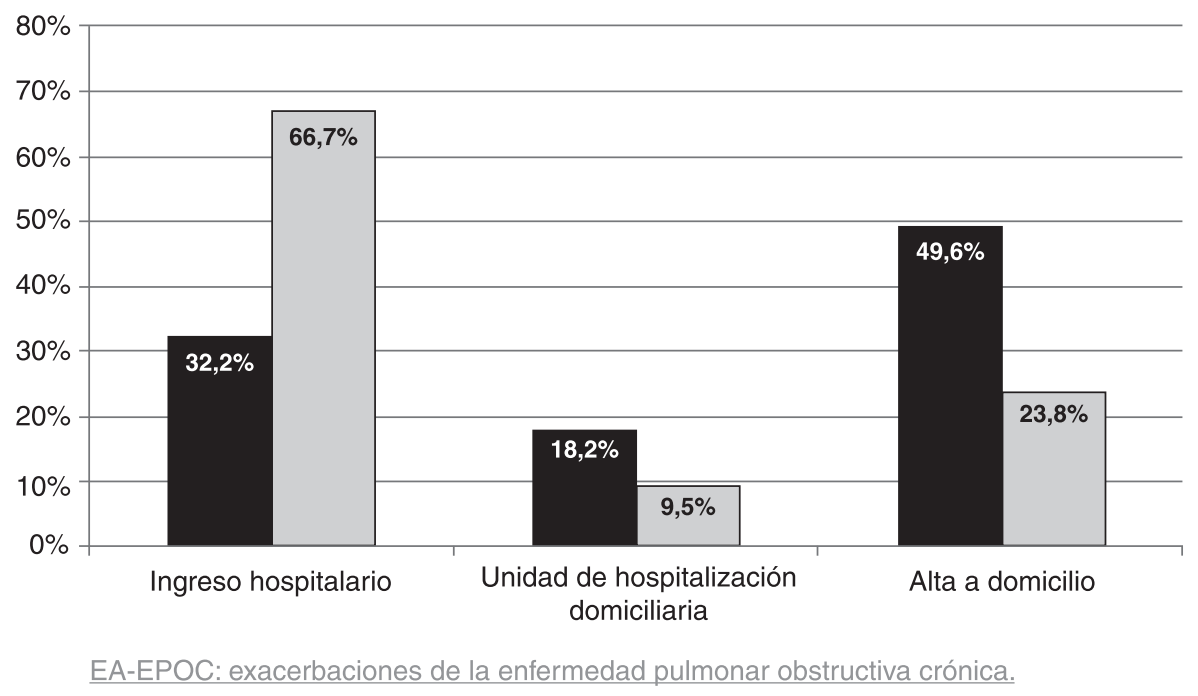

Figura 1. Destino final de los pacientes atendidos por exacerbación de la enfermedad pulmonar obstructiva crónica.

mayor tasa de ingresos por EA-EPOC, destacando el porcentaje del valor predicho de $\mathrm{FEV}_{1}$ y la adecuación de la prescripción de oxigenoterapia crónica domiciliaria (OCD). En nuestro estudio no encontramos diferencias en la gravedad basal de la EPOC entre los dos grupos, pero sí en cuanto al porcentaje de pacientes portadores de OCD, sin poder evaluarse la adecuación de la misma.

Los HD fueron creados a partir de la necesidad de ofrecer tratamientos y procedimientos diagnósticos frecuentes a pacientes con diferentes patologías que así lo precisaran. En nuestro caso, el HDER surgió con la intención de proporcionar a los pacientes con EPOC grave un mecanismo de atención periódica apoyado en un programa de atención continuada (PAC) con el objetivo de reducir las exacerbaciones y sus consecuencias sobre el curso de la enfermedad. Dicho programa, entre otros, incluye: (a) control médico periódico, (b) control preferente tras las exacerbaciones u hospitalización, (c) educación sanitaria, (d) programa de fisioterapia y (e) deshabituación tabáquica. Así mismo, en el caso específico de los pacientes con EPOC grave o muy grave (estadios GOLD III-IV),el principal criterio de inclusión en el PAC es la presencia de 2 o más exacerbaciones de la enfermedad en el último año que hayan requerido ingreso hospitalario o atención en urgencias ${ }^{6}$. La inclusión, control, educación y seguimiento de este grupo de pacientes ha permitido alcanzar importantes índices de control de la enfermedad y de conocimiento de los mismos, tanto desde el punto de vista clínico (situación basal, perfil psicológico y de cumplimiento) como sociofamiliar, favoreciendo que en las exacerbaciones sea posible establecer, considerando dichos factores, quiénes son tributarios de tratamiento ambulatorio o en régimen de hospitalización domiciliaria o bien de ingreso en planta convencional.

Otro de los resultados destacables de nuestro trabajo es la hospitalización domiciliaria de los pacientes con EA-EPOC, una alternativa altamente evaluada y reconocida, y con un claro impacto económico (reducción de costes) y sobre la dinámica hospitalaria (disponibilidad de camas), sin comprometer la satisfacción ni la garantía del cuidado del paciente ${ }^{9,10}$. En nuestro estudio se observa una diferencia claramente significativa en la utilización de esta modalidad de tratamiento domiciliario en el grupo de pacientes atendidos en el HDER, lo cual podría explicarse por una elección de los pacientes candidatos basada en un mayor 
conocimiento de las condiciones domiciliarias y del cumplimiento ambulatorio.

Entre las limitaciones del estudio, habría que destacar que no se puede descartar completamente que no exista un sesgo en la selección de los pacientes. Sin embargo, en lo referente a las características basales, ambos grupos eran comparables en cuanto a la comorbilidad (índice de Charlson) y a la gravedad de la EPOC en situación de estabilidad (medida por el $\mathrm{FEV}_{1}$ ) y, de hecho, los resultados de las otras variables evaluadas muestran en cualquier caso mayor gravedad en los pacientes atendidos en el HDER (mayor número de ingresos en el año previo y número superior de pacientes en situación de insuficiencia respiratoria crónica).

De la misma manera, en lo relacionado con la gravedad de la exacerbación, tampoco se encontraron diferencias entre los dos grupos, aunque cabe tener en consideración que actualmente no se dispone de una escala bien definida para establecer la gravedad en las EA-EPOC. Así mismo, a lo largo de todo el estudio fue difícil evaluar datos objetivos inherentes a la EA-EPOC en todos los pacientes (por ejemplo frecuencia respiratoria y gasometría arterial). De todas formas, creemos que la realización de un análisis multivariado permite ajustar las diferencias entre los dos grupos.

Finalmente, otro de los elementos a tener en cuenta es que el ámbito donde se atiende al paciente (HDER o UCIAS) influya por sí mismo, por su propio modelo organizativo, en el destino final del paciente.

En conclusión, la atención de los pacientes con EA-EPOC en un HDER por un equipo médico y de enfermería y fisioterapia, que además los controla de forma habitual en situación estable, parece reducir el número de ingresos hospitalarios en comparación con la atención convencional en un Servicio de Urgencias.

\section{Financiación}

Este trabajo ha sido financiado parcialmente por el Fondo de Investigación Sanitaria (FIS 06/90282, FIS 06/0326).

\section{Conflicto de intereses}

Los autores declaran no tener ningún conflicto de intereses.

\section{Agradecimientos}

Este trabajo ha sido posible gracias a la inestimable colaboración de la Sra. Nuria Torà, y de todo el personal de enfermería del Hospital de día de Neumología y del Servicio de Urgencias del Hospital de Sabadell, a quienes agradecemos su inagotable capacidad de trabajo y generosidad.

\section{Bibliografía}

1. Wang Q Bourbeau J. Outcomes and health-related quality of life following hospitalization for an acute exacerbation of COPD. Respirology. 2005;10: 334-40.

2. Otero González I, Blanco Aparicio M, Montero Martínez C, Valiño López P, Verea Hernando $\mathrm{H}$. Características epidemiológicas de las exacerbaciones por EPOC y asma en un hospital general. Arch Bronconeumol. 2002;38:256-62.

3. Miravitlles M, Figueras M. El coste de la enfermedad pulmonar obstructiva crónica en España. Opciones para una optimización de recursos. Arch Bronconeumol. 2001;37:388-93.

4. Domingo Ch, Sans-Torres J, Solà J, Espuelas H, Marín A. Efectividad y eficiencia de una consulta monográfica hospitalaria para pacientes con EPOC e insuficiencia respiratoria. Arch Bronconeumol. 2006;42:104-12.

5. Solera JJ, Martínez-García MA, Román P, Orero R, Terrazas S, Martínez-Pechuán A. Eficacia de un programa específico para pacientes con EPOC que presentan frecuentes agudizaciones. Arch Bronconeumol. 2006;42:501-8.

6. Pomares Amigó X, Montón Soler C. Hospitales de día de enfermedades respiratorias: ¿qué hemos aprendido? Med Clin (Barc). doi:10.1016/j.medcli.2009.07.028

7. Peces-Barba G, Barberà JA, Agustí A, Casanova C, Casas A, Izquierdo JL, et al. Guía clínica SEPAR-ALAT de diagnóstico y tratamiento de la EPOC. Arch Bronconeumol. 2008;44:271-81.

8. García-Aymerich J, Monsó E, Marrades RM, Escarrabill J, Félez MA, Sunyer J, et al. Risk factors for hospitalization for a chronic obstructive pulmonary disease exacerbation. EFRAM Study. Am J Respir Crit Care Med. 2001;164:1002-7.

9. Puig-Junoy J, Casas A, Font-Planells J, Escarrabill J, Hernández C, Alonso J, et al. The impact of home hospitalization on healthcare costs of exacerbations in COPD patients. Eur J Health Econ. 2007;8:325-32.

10. Díaz Lobato S, González Lorenzo F, Gómez Mendieta MA, Mayorales Alises S, Martín Arechabala I, Villasante Fernández-Montes C. Evaluación de un programa de hospitalización domiciliaria en pacientes con EPOC agudizada. Arch Bronconeumol. 2005;41:5-10. 
Bellaterra, 31 de octubre de 2012

A quien corresponda

Como uno de los autores del artículo "HOSPITAL DE DÍA DE ENFERMEDADES RESPIRATORIAS: IMPACTO SOBRE LA TASA DE INGRESOS HOSPITALARIOS POR EXACERBACIONES DE LA ENFERMEDAD PULMONAR OBSTRUCTIVA CRÓNICA", publicado en la revista MEDICINA CLÍNICA [Ref.: Med Clin (Barc). 2011;136(15):665-668], autorizo a que el Lic. LUIS FERNANDO CASAS MÉNDEZ, lo utilice como TRABAJO DE INVESTIGACIÓN en el programa de Doctorado del Departament de Medicina de la Universitat Autònoma de Barcelona.

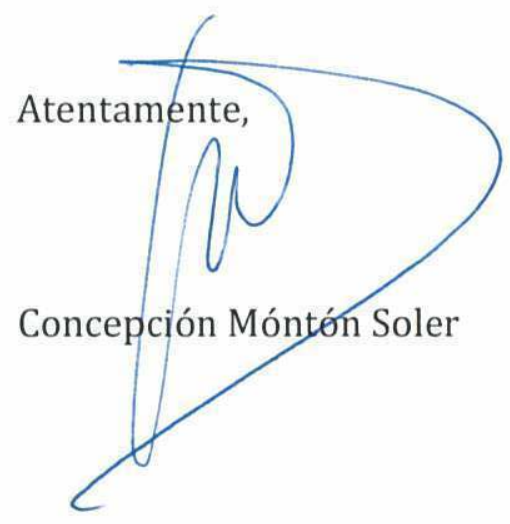


Bellaterra, 31 de octubre de 2012

A quien corresponda

Como uno de los autores del artículo "HOSPITAL DE DÍA DE ENFERMEDADES RESPIRATORIAS: IMPACTO SOBRE LA TASA DE INGRESOS HOSPITALARIOS POR EXACERBACIONES DE LA ENFERMEDAD PULMONAR OBSTRUCTIVA CRÓNICA", publicado en la revista MEDICINA CLÍnICA [Ref.: Med Clin (Barc). 2011;136(15):665-668], autorizo a que el Lic. LUIS FERNANDO CASAS MÉNDEZ, lo utilice como TRABAJO DE INVESTIGACIÓN en el programa de Doctorado del Departament de Medicina de la Universitat Autònoma de Barcelona.

Atentamente,

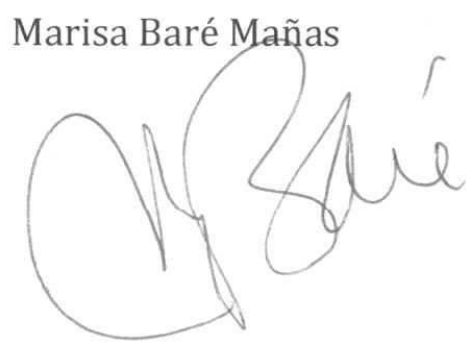


Bellaterra, 31 de octubre de 2012

A quien corresponda

Como uno de los autores del artículo "HOSPITAL DE DÍA DE ENFERMEDADES RESPIRATORIAS: IMPACTO SOBRE LA TASA DE INGRESOS HOSPITALARIOS POR EXACERBACIONES DE LA ENFERMEDAD PULMONAR OBSTRUCTIVA CRÓNICA", publicado en la revista MEDICINA CLÍNICA [Ref.: Med Clin (Barc). 2011;136(15);665-668), autorizo a que el Lic. LUIS FERNANDO CASAS MÉNDEZ, lo utilice como TRABAJO DE INVESTIGACIÓN en el programa de Doctorado del Departament de Medicina de la Universitat Autònoma de Barcelona.

Atentamente,

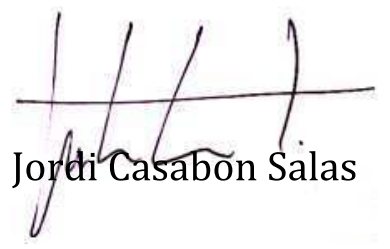


Bellaterra, 31 de octubre de 2012

A quien corresponda

Como uno de los autores del artículo "HOSPITAL DE DÍA DE ENFERMEDADES RESPIRATORIAS: IMPACTO SOBRE LA TASA DE INGRESOS HOSPITALARIOS POR EXACERBACIONES DE LA ENFERMEDAD PULMONAR OBSTRUCTIVA CRÓNICA", publicado en la revista MEDICINA CLÍNICA [Ref.: Med Clin (Barc). 2011;136(15):665-668], autorizo a que el Lic. LUIS FERNANDO CASAS MÉNDEZ, lo utilice como TRABAJO DE INVESTIGACIÓN en el programa de Doctorado del Departament de Medicina de la Universitat Autònoma de Barcelona.

Atentamente,

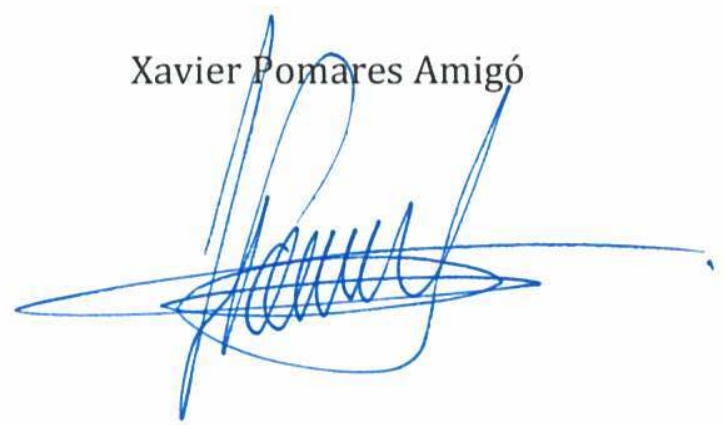


A quien corresponda

Como uno de los autores del artículo "HOSPITAL DE DÍA DE ENFERMEDADES RESPIRATORIAS: IMPACTO SOBRE LA TASA DE INGRESOS HOSPITALARIOS POR EXACERBACIONES DE LA ENFERMEDAD PULMONAR OBSTRUCTIVA CRÓNICA", publicado en la revista MEDICINA CLÍNICA [Ref.: Med Clin (Barc). 2011;136(15);665-668), autorizo a que el Lic. LUIS FERNANDO CASAS MÉNDEZ, lo utilice como TRABAJO DE INVESTIGACIÓN en el programa de Doctorado del Departament de Medicina de la Universitat Autònoma de Barcelona.

Atentamente,

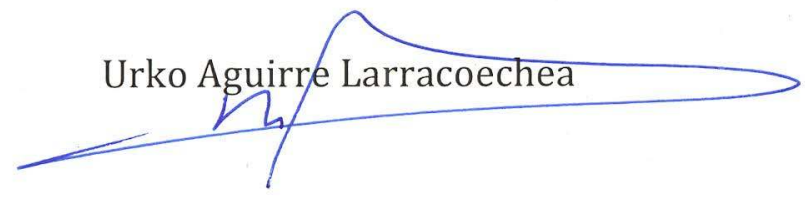

